\title{
A Solution of Boussinesq's equation for infiltration phenomenon in unsaturated porous media by Homotopy Analysis Method
}

\author{
Kajal K. Patel ${ }^{1}$, M. N. Mehta ${ }^{2}$, Twinkle R. Singh ${ }^{3}$ \\ ${ }^{I}$ (Research Scholar, S. V. National Institute of Technology, Surat-395007, India, \\ ${ }_{2}^{2}$ (Professor and Head of Department of Applied Mathematics \& Humanities, S. V. National Institute of \\ Technology, Surat-395007, India, \\ ${ }^{3}$ (Assistant Professor in Department of Applied Mathematics \& Humanities, S. V. National Institute of \\ Technology, Surat-395007, India,
}

\begin{abstract}
The nonlinear Boussinesq's equation for infiltration phenomenon in unsaturated porous media is nonlinear partial differential equation and it has been solved by using Homotopy analysis method. the solution gives height of free surface of infiltrated water mound in unsaturated porous media by using appropriate initial guess value of the solution. The solution is physically interpreted and it is concluded that the height of free surface of infiltrated water in unsaturated porous media is decreasing and graph of the solution is given by using Maple coding.
\end{abstract}

Keywords: - Boussinesq's equation, Unsaturated porous media, Homotopy analysi Method, Infiltration

\section{INTRODUCTION}

The unsaturated zone is distinctly involved in many aspect of hydrology, infiltration, evaporation, groundwater recharge, soil moisture storage and soil erosion. The infiltration process is one of a far larger class of problems involving fluid flow in porous media. The infiltration of fluid into the pores of unsaturated porous media (soil) is played an important role in various fields: science and engineering, including hydrogeology, chemical engineering, agriculture engineering, reservoir science, fluid mechanics, environmental engineering and biomechanics [1], [2], [16].

In an environment, many phenomena that govern infiltration processes have been extensively analyzed and compared with experimental results. The infiltration phenomenon is useful to control salinity of water, contamination of water, radioactive waste deposit and agriculture purpose such problems are also useful to measure moisture content of water in vertical one-dimension ground water recharge and dispersion of any fluid in porous media. The rate of the movement of groundwater is called infiltration rate. The rate of infiltration normally decreases as the soil becomes saturated [8]. Infiltration is governed by, gravity, Capillary action and soil porosity. While smaller pores offer greater resistance to gravity, very small pores pull water through Capillary action, in addition to and even against, the force of gravity [2]. Soil porosity plays an important role in the movement of groundwater into unsaturated porous media (soil). Infiltrated groundwater fills the unsaturated soil and reduces the capillary forces drawing water into the pores. As the soil saturates, the micelle structure of the clay particles absorbs water causing them to expand. This expansion reduces the size of soil pores. V'azquez [18] reported that the present model was developed first by Boussineq in 1903 [3]. In 1972, Bear [2] explained that atmospheric pressure in the dry region by using the relation between pressure and height of free surface and velocity of infiltrated water can be calculated by Darcy's law [4]. Infiltration phenomenon has been discussed by different authors from different viewpoints in homogeneous porous media as well as heterogeneous porous media by Patel and Mehta[15], Desai \& Mehta [5], and Patel \& Mehta [14]. Study of liquid infiltration into pours media would of significance interest in understanding the process related to hydraulic fracturing of pours media due to escape of entrapped hydrocarbon gasses at very high pressure. This has been evident in the interactions of rainfall preserved to be a major factor in the collapse of the volcanic lava domes collapses at the Soufriere Hills Volcano Montserrat [17].

Based on the Homotopy method in topology, Liao proposed such a new kind of analytic technique, known as Homotopy analysis method. The Homotopy analysis method has a great advantage that, in general its validity does not depend upon on small or large parameters, and it is easy to adjust the convergence region and rate of approximation series. Therefore, the Homotopy analysis method handles linear and nonlinear problems without any assumption and restriction. We note here that Homotopy perturbation method [10], is only a special case of the Homotopy Analysis method [10]. Indeed Liao [10], [11] makes a compelling case that the Adomain decomposition method, The Lyapunov artificial small parameter method and the $\delta$-expansion method are nothing but special cases of the Homotopy Analysis method. Using one interesting property of Homotopy, we can transform any non-linear problem into linear problems. Homotopy Analysis method has been applied to 
nonlinear fluid dynamics problems [11]. The Homtopy Analysis method contains the auxiliary parameter $\hbar$, which provides over here a simple way to adjust and control the convergence region of solution series for large values of $t$ [9].

The present paper discusses the approximate analytical solution of nonlinear partial differential equation for infiltration phenomenon, to examine the height of the free surface of the water mound in unsaturated homogeneous soil. The changes in saturated porous media and distribution of pore pressures can be calculated by using the Homotopy Analysis Method. Its solution gives the height of the free surface of the water mound in soil at any distance $\mathrm{X}$ at time $\mathrm{T}>0$. The graph of height of infiltrated groundwater verses time $\mathrm{T}$ are given at distance $\mathrm{X}$, which shows at any distance $\mathrm{X}$ height of infiltrated groundwater decreases as time $\mathrm{T}$ increases.

\section{STATEMENT OF THE PROBLEM}

The reservoir field with water of height $\mathrm{OA}=h_{\max }=$ maximum height with impermeable bottom and surrounding of this reservoir is unsaturated homogeneous soil. Infiltration phenomenon is well demonstrated in the following figure 1that shows a cross section of the reservoir surrounded by unsaturated porous medium.

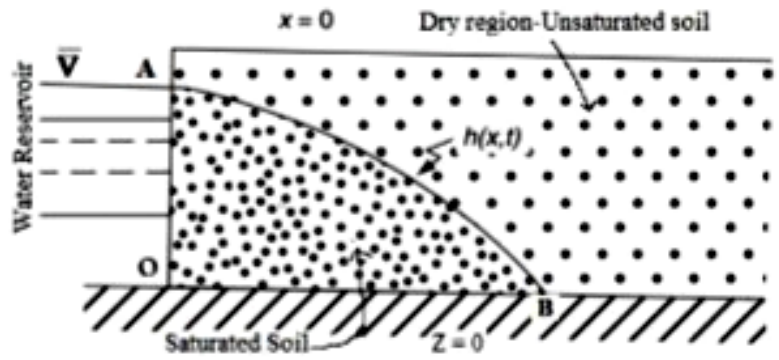

Figure 1: A scheme of groundwater Infiltration.

The height of the free surface is zero, when $\mathrm{OB}=\mathrm{x}=1$, the dotted arc below the curve is saturated by infiltrated groundwater and above the curve is the dry region of unsaturated soil. The bottom is assumed impermeable, so water cannot flow in a downward direction. Infiltration is the process by which the groundwater of the reservoir has entered into the unsaturated soil through vertical permeable wall. The groundwater infiltrated in an unsaturated soil; its velocity decreases as soil becomes saturated. The infiltrated groundwater will enter in unsaturated soil then the infiltrated groundwater will develop a curve between saturated soil and unsaturated soil, which is called a water table or water mound. To understand this onedimensional infiltration phenomenon and for the sake of its mathematical formulation, we have taken some assumptions. The governing nonlinear partial differential equation for the height of infiltrated water is known as Boussinesq's equation [3]. The purpose of the study of infiltration is to determine the effective height of the free surface as a measure of initial storage capacity of a porous stratum.

\section{MATHEMATICAL FORMULATION}

Consider the maximum height $h_{\max }$ of the groundwater reservoir and adjacent formation of the reservoir is unsaturated porous media shown in the figure 1 by dotted region. The groundwater is infiltrated through the vertical adjacent side with the following simplifying assumptions:

1. The stratum has height $h_{\max }$ and lies on top of a horizontal impervious bed, which we label as $\mathrm{z}=0$;

2. We ignore the transversal variable y;

3. The water mass which infiltrates the soil occupies a region described as

$$
\Omega=\{(x, z) \in R: z \leq h(x, t)\}
$$

We are assuming that there is no region of partial saturation. This is an evolution model. Clearly, $0 \leq h(x, t) \leq h_{\max }, h_{\max }$ maximum height of free surface and the free boundary function ' $\mathrm{h}$ ' is also an unknown function for this problem. In this situation, we arrive at a system of three equations with unknowns the two velocity components ' $u$ ', ' $w$ ' and ' $p$ ' in a variable domain: one equation of mass conservation for an incompressible fluid and two equations for the conservation of momentum of the Navier-stokes type.

The resulting system is too complicated and can be simplified for the practical computation after introducing a suitable assumption, the hypothesis of almost horizontal flow, i.e. assuming that an almost horizontal flow where speed $u \square(u, 0)$,so that 'h' has small gradients. Hence vertical component of the momentum equation can be written as; 
$p\left(\frac{d u_{z}}{d t}+u . \nabla u_{z}\right)=-\frac{\partial p}{\partial z}-\rho g$

The inertial term has been neglected (the left-hand side).

Integration equation (1) with respect to $\mathrm{z}$ gives for the first approximation, it gives

$p+\rho g z=$ cons $\tan t$

We now calculate the constant on the free surface $z=h(x, t)$. If we impose continuity of the pressure across the interface, we have $\mathrm{p}=0$ (assuming constant atmospheric pressure in the air that fills the pores of the dry region $\mathrm{z}>\mathrm{h}(\mathrm{x}, \mathrm{t}))$. We then get

$p=\rho g(h-z)$

(3)

Hence the pressure can be established using of the hydrostatic approximation. We go now to the mass conservation law which will give us the equation. We proceed as follows: let us take a section $S=(x, x+a) \times(0, C)$. Then

$\phi \frac{\partial}{\partial t} \int_{x}^{x+a} \int_{0}^{h} d y d x=-\int_{\partial s} u . n d l$

where ' $\phi$ ' is the porosity of the medium, i.e., the fraction of volume available for the flow circulation, and ' $u$ ' is the seepage velocity, which obeys Darcy's law in the form that includes gravity effects[4],

$u=-\frac{k}{\mu} \nabla(p+\rho g z)$

On the right-hand lateral surface, we have $u \cdot n \approx(u, 0) \cdot(1,0)=u$, i.e. $\left(\frac{k}{\mu}\right) p_{x}$, while, on the left-hand side, we have the seepage velocity as '-u'.

Using the formula for ' $p$ ' and differentiating with respect to ' $x$ ', we get

$\phi \frac{\partial h}{\partial t}=\frac{p g K}{\mu} \frac{\partial}{\partial x} \int_{0}^{h} \frac{\partial}{\partial x} h d z$

Thus we obtained Boussinesq's equation as[3],

$\frac{\partial h}{\partial t}=\frac{p g K}{2 \mu \phi} \frac{\partial^{2}}{\partial x^{2}}\left(h^{2}\right)$

with constant $\beta=\frac{p g K}{2 \mu \phi}$ this is the fundamental equation in ground water infiltration which respects porous medium equation. On choosing new dimensionless variable $T=\frac{p g K}{\phi \mu} t$ and $X=\frac{x}{L}$. In equation (7), It gives, $\frac{\partial h}{\partial T}=\left\{h \frac{\partial^{2} h}{\partial X^{2}}+\left(\frac{\partial h}{\partial X}\right)^{2}\right\}$

The equation (8) gives the height of the water mound with initial and boundary condition as,

$h(0, T)=h_{\max } \quad X=0$ and $T>0$

which satisfied the initial condition

$h(\mathrm{X}, 0)=\mathrm{h}_{0} \quad X>0$ and $T=0$.

Equation (8) is a fundamental equation in groundwater infiltration. Once $h(\mathrm{X}, \mathrm{T})$ is calculated, we may calculate the pressure via (2) and then the speed by means of Darcy's law.

Using the transformation $\frac{h(X, T)}{h_{0}}=H(X, T)$ and considering $h_{0}{ }^{2}=\varepsilon$, Equation (8) becomes, 


$$
\frac{\partial H}{\partial T}=\left\{\varepsilon H \frac{\partial^{2} H}{\partial X^{2}}+\left(\frac{\partial H}{\partial X}\right)^{2}\right\}
$$

with appropriate guess value of the solution as [12],

$h(\mathrm{X}, \mathrm{T}: \varepsilon)=\left(h_{\max }-\frac{1}{2} T X^{2}\right) e^{-X}+\varepsilon^{m}$

which satisfy the initial and boundary condition of equation (8).

\section{THE SOLUTION WITH HOMOTOPY NALYSIS METHOD}

For one dimensional non-linear partial differential equation for infiltration phenomenon, we assume that the $h_{\max }$ is initial height of infiltrated groundwater through the pores of an unsaturated soil, at time $\mathrm{T}=0$ is expressed as,

$h(X, T, \varepsilon=0)=\left(h_{\max }-\frac{1}{2} T X^{2}\right) e^{-X}+\varepsilon^{m}$,

where $\varepsilon=0$ for time $\mathrm{T}=0$, the height of infiltrated water in unsaturated soil.

Now we apply the Homotopy analysis method into the infiltration phenomenon for unsaturated homogeneous soil. We consider the equation (8) as nonlinear partial differential equation as

$\square[h(X, T ; \varepsilon)]=0$

Where $\square$ is a non-linear operator, $h(X, T ; \varepsilon)$ is considered as unknown function which represent the height of free surface for infiltrated groundwater through the pores of an unsaturated soil at any distance $X$ for given time $\mathrm{T}>0$, for $0 \leq \varepsilon \leq 1$. We use auxiliary linear operator $\mathfrak{J}[h(X, T ; \varepsilon)]=\frac{\partial h(X, T ; \varepsilon)}{\partial T}$ and initial approximation of the height of free surface for infiltrated groundwater into soil $H_{0}(X, T)=\left(h_{\max }-\frac{1}{2} T X^{2}\right) e^{-X}$ to construct the corresponding zero ${ }^{\text {th }}$ order deformation equation. As the auxiliary linear operator $\mathfrak{I}$ which satisfies $\mathfrak{I}\left[C_{4}\right]=0$, where $C_{4}$ is arbitrary constant. This provides a fundamental rule to direct the choice of the auxiliary function $H(X, T) \neq 0$, the initial approximation $H_{0}(X, T)$, and the auxiliary linear operator $\mathfrak{I}$, called the rule of solution expression. Establish the zero-order deformation equation of infiltration phenomenon as [9],

$(1-\varepsilon) \mathfrak{I}\left[h(X, T ; \varepsilon)-H_{0}(X, T)\right]=\varepsilon \hbar H(X, T) \square[h(X, T ; \varepsilon)]$

where $H_{0}(X, T)$ denote an initial guess value of the height free surface (infiltration) into the unsaturated soil of the exact solution $H(X, T)$ which is our purpose to find it. Since $\hbar \neq 0$ is an auxiliary parameter and $H(X, T) \neq 0$ is an auxiliary function such that $\varepsilon \in[0,1]$ is an embedding parameter. The auxiliary parameter $\hbar$ is providing a simple way to ensure the convergence of series. Thus it renamed $\hbar$ as convergence control parameter [9]. Let $\mathfrak{I}$ is an auxiliary linear operator with the property that,

$\mathfrak{I}[h(X, T ; \varepsilon)]=0$ when $h(X, T ; \varepsilon)=0$

When $\varepsilon=0$, the zero-order deformation equation (15) becomes

$\mathfrak{I}\left[h(X, T ; \varepsilon)-H_{0}(X, T)\right]=0$

Which gives the first rule of solution expression and according to the initial guess $H_{0}(X, T)=\left(h_{\max }-\frac{1}{2} T X^{2}\right) e^{-X}$, it is straightforward to choose

$h(X, T ; 0)=H_{0}(X, T)$ 
When $\varepsilon=1$, since $\hbar \neq 0, H(X, T) \neq 0$ the zero-order deformation equation (11) is equivalent to

$$
\square[h(X, T ; \varepsilon)]=0
$$

which is exactly the same as the original equation (14) provided

$$
h(X, T ; 1)=H(X, T)
$$

According to (17) and (19) as the embedding parameter $\varepsilon$ increases from 0 to 1 , solution $h(X, T ; \varepsilon)$ varies continuously from the initial guess value of the height free surface $H_{0}(X, T)$ of an unsaturated soil to the solution $H(X, T)$ and its solution is assumed by expanding $h(X, T ; \varepsilon)$ in Taylor series with respect to $\varepsilon$ as,

$$
h(X, T ; \varepsilon)=h(X, T ; 0)+\sum_{m=1}^{\infty} H_{m}(X, T) \varepsilon^{m}
$$

Where, $H_{m}(X, T)=\left.\frac{1}{m !} \frac{\partial^{m} h(X, T ; \varepsilon)}{\partial \varepsilon^{m}}\right|_{\varepsilon=0}$

i.e. the height of free surface is the function of distance $\mathrm{X}$ and time $\mathrm{T}$ for any parametric value $\varepsilon$ is expressed as, the infiltration of groundwater at time $T=0, H_{0}(X, T)$ and sum of infiltration of groundwater into the unsaturated soil $H_{1}(X, T), H_{2}(X, T), \ldots$ at different time $\mathrm{T}$ for different value of parameter $\varepsilon$. Here, the series (20) is called homotopy-series; the series (21) is called homotopy series solution of $\square[h(X, T ; \varepsilon)]=0$ and $H_{m}(X, T)$ is called the $\mathrm{m}^{\text {th }}$-order derivative of $h$. Auxiliary parameter $\hbar$ in Homotopy-series (20) can be regard as iteration factor and is widely used in numerical computations. It is well known that the properly chosen iteration factor can ensure the convergence of Homotopy series (20) is depending upon the value of $\hbar$, one can ensure that convergent of Homotopy series, solution simply by means of choosing the proper value of $\hbar$ as shown by Liao $[9,10,11,12]$. If the auxiliary linear operator, the initial guess, the auxiliary parameter $\hbar$, the auxiliary function $H(X, T)$ are so properly chosen, the series (20) converges at $\varepsilon=1$.

Hence the height of infiltrated groundwater can be expressed as,

$$
H(X, T)=H_{0}(X, T)+\sum_{m=1}^{\infty} H_{m}(X, T)
$$

And $H_{m}(X, T)$ can be calculated by equation (27). This must be one of the solution of original non-linear partial differential equation (11) the height of infiltration groundwater problem in unsaturated homogenous soil. According to the definition (21), the governing equation can be deduced from the zero-order deformation equation (15), define the vector

$$
\vec{H}_{m}=\left\{H_{0}(X, T), H_{1}(X, T), \ldots, H_{n}(X, T)\right\}
$$

Differentiating equation (15) m-times with respect to the embedding parameter $\varepsilon$ and then setting $\varepsilon=0$ and finally dividing them by $m$ !, we have the so-called $\mathrm{m}^{\text {th }}$ order deformation equation of the height of infiltration groundwater $H(X, T)$ will be as, [9]

$$
\mathfrak{I}\left[H_{m}(X, T)-\chi_{m} H_{m-1}(X, T)\right]=\varepsilon \hbar H(X, T) R_{m}\left(\vec{H}_{m-1}, X, T\right)
$$

Where $R_{m}\left(\vec{H}_{m-1}, X, T\right)=\left.\frac{1}{(m-1) !} \frac{\partial^{m-1} \square[h(X, T ; \varepsilon)]}{\partial \varepsilon^{m-1}}\right|_{\varepsilon=0}$

And $\chi_{m}= \begin{cases}0, & m \leq 1 \\ 1, & m>1\end{cases}$ 
It should be emphasized that $H_{m}(X, T)$ for $m \geq 1$, is governed by the linear equation (23) with the linear boundary condition that came from the original problem, which can solve by symbolic computation software Maple as bellow. The rule of solution expression as given by equation (10) and equation (15), the auxiliary function independent of $\varepsilon$ can be chosen as $H(X, T)=1$ [9].

According to (19) and taking inverse of equation (23) the equation (24) becomes,

$$
\begin{aligned}
& H_{m}(X, T)=\chi_{m} H_{m-1}(X, T)+\hbar \mathfrak{I}^{-1}\left[R_{m}\left(\vec{H}_{m-1}, X, T\right)\right] \\
& R_{m}\left(\vec{H}_{m-1}, X, T\right)=\left.\frac{1}{(m-1) !} \frac{\partial^{m-1} \square[h(X, T ; \varepsilon)]}{\partial \varepsilon^{m}}\right|_{\varepsilon=0}
\end{aligned}
$$

In this way, we get $h_{m}(X, T)$ for $\mathrm{m}=1,2,3, \ldots$ successively by using Maple software as,

$$
\begin{aligned}
& H_{1}(X, T)=-\frac{1}{6} \hbar T\left(\begin{array}{l}
3 T^{2} X^{2}-4 T^{2} X^{3}+T^{2} X^{4}-2.94 T \\
+11.76 T X-5.88 T X^{2}+3 X^{2} e^{X}+11.5248
\end{array}\right) e^{-2 X} \\
& H_{2}(X, T)=-\frac{1}{240} T h\left(\begin{array}{l}
1117.20 X T^{3} h+470.40 T X e^{X}-235.20 T h e^{X}-234.20 T X^{2} e^{X} \\
-3226.9440 X T^{2} h+144 X^{2} T^{4} h-288 X^{5} T^{4 h}+36 X^{6} T^{4} h-608 X^{3} T^{4} h \\
+708 X^{4} T^{4} h+1382.9760 T^{2} h X^{2}+360 X^{2} T^{2} h e^{X}+120 X^{4} T^{2} h e^{X} \\
-480 X^{3} T^{2} h e^{X}-160 T^{2} X^{3} e^{X}+40 T^{2} X^{4} e^{X}+120 X^{2} e^{2 X}+120 X^{2} h e^{2 X} \\
-117.60 T e^{X}+460.9920 h e^{X}-2032.974720 T h-88.20 T^{3} h \\
+1575.056 T^{2} h+460.992 e^{X}-470.40 X^{2} T h e^{X}+940.80 X T h e^{X} \\
+120 T^{2} X^{2} e^{X}-352.80 T^{3} h X^{4}+1822.80 T^{3} h X^{3}-2631.30 T^{3} h X^{2}
\end{array}\right) e^{-3 X}
\end{aligned}
$$

Using initial guess value of height of the infiltration groundwater into the soil equation (12) and successive

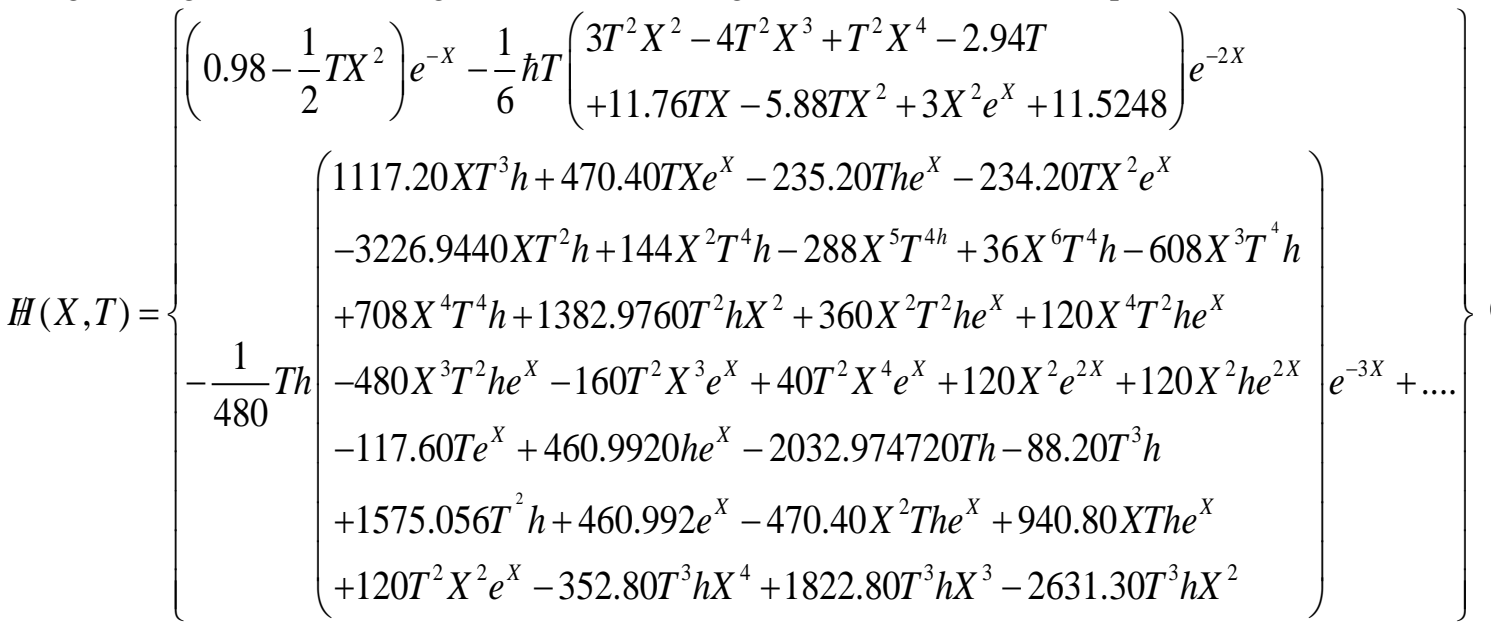

\section{NUMERICAL AND GRAPHIAL SOLUTION}

Numerical and graphical presentations of equation (30) have been obtained by using Maple coding. Figure 2 represents the graphs of infiltrated groundwater $H(X, T)$ vs. distance $\mathrm{X}$, and time $T=0.1,0.2,0.3$, $0.4,0.5$ is fixed, figure 3 represents the graphs of infiltrated groundwater $H(X, T)$ vs. distance $\mathrm{X}$, and time $T$ $=0.6,0.7,0.8,0.9,1.0$ is fixed and Table I indicate the numerical values of height of infiltrated groundwater for different time $\mathrm{T}$ and distance $\mathrm{X}$. The figure $2 \& 3$, and the table 1 indicates the graphical representations of the infiltration phenomenon in homogeneous soil. The convergence of the Homotopy series (18) is dependent upon the value of convergence-parameter $\hbar[6,9,10,17]$. Therefore, we choose the proper value of the convergenceparameter $\hbar=0.1$ to obtain convergent Homotopy-series solution [9]. 
A Solution of Boussinesq's equation for infiltration phenomenon in unsaturated porous media by

TABLE I : Height of infiltrated groundwater $H(X, T)$ for different time $\mathrm{T}$ for fixed distance $\mathrm{X}=0.1,0.2,0.3$, $0.4,0.5,0.6,0.7,0.8,0.9,1.0$.

\begin{tabular}{|c|c|c|c|c|c|c|c|c|c|c|}
\hline Distance & \multicolumn{10}{|c|}{ Height of infiltrated groundwater } \\
\cline { 2 - 10 } $\mathrm{X}$ & $\mathrm{T}=\mathbf{0 . 1}$ & $\mathrm{T}=\mathbf{0 . 2}$ & $\mathrm{T}=\mathbf{0 . 3}$ & $\mathrm{T}=\mathbf{0 . 4}$ & $\mathrm{T}=0.5$ & $\mathrm{~T}=\mathbf{0 . 6}$ & $\mathrm{T}=0.7$ & $\mathrm{~T}=\mathbf{0 . 8}$ & $\mathrm{T}=\mathbf{0 . 9}$ & $\mathrm{T}=1.0$ \\
\hline 0.1 & 0.8587 & 0.8326 & 0.8082 & 0.7852 & 0.7425 & $\mathbf{0 . 7 4 2 5}$ & $\mathbf{0 . 7 2 2 4}$ & $\mathbf{0 . 7 0 2 9}$ & $\mathbf{0 . 6 8 3 8}$ & $\mathbf{0 . 6 6 4 9}$ \\
\hline 0.2 & 0.7777 & 0.7539 & 0.7314 & 0.7095 & 0.6675 & $\mathbf{0 . 6 6 7 5}$ & 0.6469 & 0.6268 & 0.6066 & 0.5863 \\
\hline 0.3 & 0.7032 & 0.6809 & 0.6593 & 0.6378 & 0.5956 & 0.5956 & 0.5746 & 0.5535 & 0.5323 & 0.5108 \\
\hline 0.4 & 0.6349 & 0.6133 & 0.5918 & 0.5703 & 0.5272 & 0.5272 & 0.5054 & 0.4835 & 0.4613 & 0.4388 \\
\hline 0.5 & 0.5726 & 0.5508 & 0.5289 & 0.5069 & 0.4625 & 0.4625 & 0.4399 & 0.4171 & 0.3939 & 0.3704 \\
\hline 0.6 & 0.5156 & 0.4932 & 0.4707 & 0.4479 & 0.4017 & 0.4017 & 0.3782 & 0.3544 & 0.3303 & 0.3059 \\
\hline 0.7 & 0.4636 & 0.4403 & 0.4168 & 0.3931 & 0.3449 & 0.3449 & 0.3205 & 0.2957 & 0.2707 & 0.2453 \\
\hline 0.8 & 0.4162 & 0.3919 & 0.3673 & 0.3425 & 0.2922 & 0.2922 & 0.2668 & 0.2409 & 0.2149 & 0.1888 \\
\hline 0.9 & 0.3731 & 0.3476 & 0.3219 & 0.2959 & 0.2435 & 0.2435 & 0.2169 & 0.1903 & 0.1635 & 0.1364 \\
\hline 1.0 & 0.3339 & 0.3073 & 0.2804 & 0.2533 & 0.1988 & 0.1988 & 0.1713 & 0.1437 & 0.1159 & 0.0881 \\
\hline
\end{tabular}

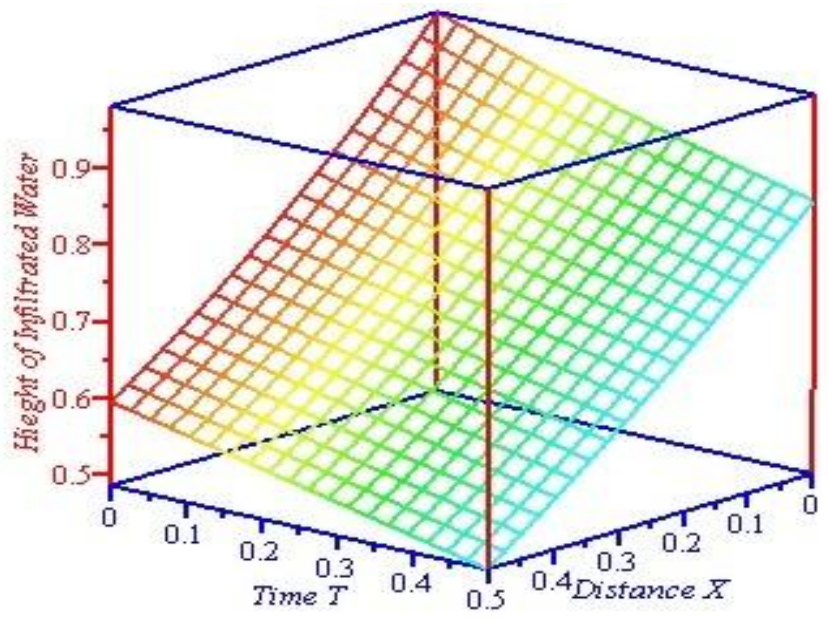

Figure 2: Represents height of infiltrated groundwater $H(X, T)$ vs. distance $\mathrm{X}$ and time $\mathrm{T}$ for auxiliary parameter $\hbar=0.1$ and auxiliary function $H(\mathrm{X}, T)=1$ [9] for $0<X<0.5$, and $0<T<0.5$

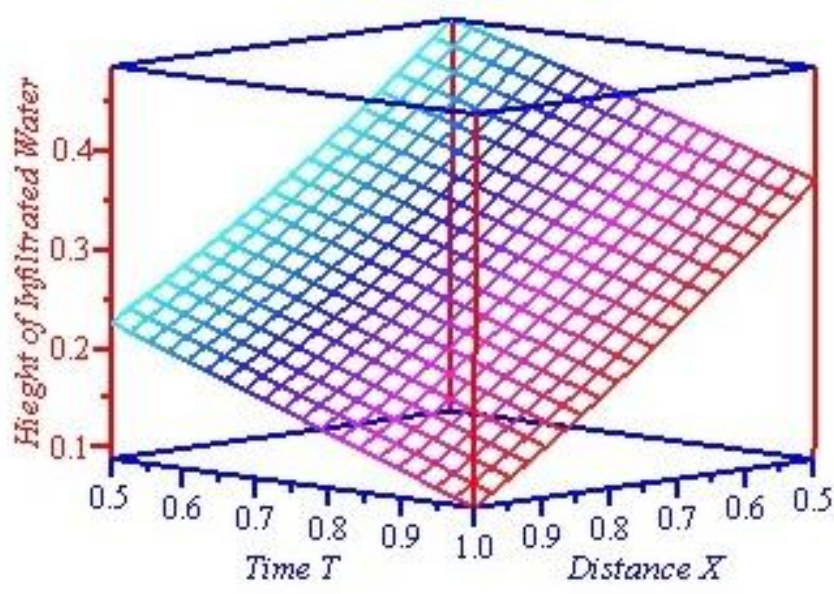

Figure 3: Represents Height of infiltrated groundwater $H(X, T)$ vs. distance $\mathrm{X}$ and time $\mathrm{T}$ for auxiliary parameter $\hbar=0.1$ and auxiliary function $H(\mathrm{X}, T)=1$ [9] for $0.5<X<1$, and $0.5<T<1$ 


\section{CONCLUSION AND DISCUSSION}

The equation (30) represents height of infiltrated groundwater $H(X, T)$ for any distance $\mathrm{X}$ and time $\mathrm{T}>0$ using Homotopy Analysis Method. It converges for embedding parameter $\varepsilon=1$ and for auxiliary parameter $\hbar=0.1$ which is expressed as negative exponential term of $\mathrm{X}$ and time $T>0$. An assumed value of infiltration $\mathrm{H}$ will be the value of the exact solution for $0<X<1$, and $0<T<1$. Figure 2 represents the height for infiltrated groundwater $H$ vs. distance $\mathrm{X}$ and time $\mathrm{T}$ for given $\mathrm{X}=0.1,0.2,0.3,0.4$ and 0.5 fixed, it shows that height of infiltrated groundwater is decreasing as distance $\mathrm{X}$ increasing for $T>0$. From figure 2, it can conclude that for $T=0.1$ infiltration if groundwater is decreasing as distance $\mathrm{X}$ increasing and when time is increasing and due to different deformation added to $H(X, T)$, the infiltration of groundwater is successively decreasing exponentially. Since the equation (11) is one-dimensional Boussinesq's equation for infiltration phenomenon, the solution is graphically as well as physically consistent with the phenomenon. Figure 3 represents the solution for infiltrated groundwater $H$ vs. distance $\mathrm{X}$ and time $\mathrm{T}$ for given $\mathrm{X}=0.5,0.6$, $0.7,0.8,0.9,1.0$. The height of the water mound is also decreasing for different time $\mathrm{T}$ for given fix value of $\mathrm{X}$. The height of infiltration of groundwater at $X=0.1$ is decreasing for different time T. After distance $\mathrm{X}$, the infiltration of groundwater is also decreasing with respect to different time $\mathrm{T}$, this resembles the scenario of $\mathrm{X}$ above. Referring both figures 2 and 3, with derived analytical result (29), it is concluded that the infiltration rate of groundwater is decreasing when distance X, as well as time, increases using Homotopy Analysis Method. This mathematical model is consistent with physical phenomenon of the infiltrated groundwater in homogeneous porous medium.

\section{REFERENCES}

[1] Bear J. and Bachmat Y., Introduction to Modeling of Transport Phenomena in Porous Media, Kluwer, Academic Publishers, Dordrecht, The Netherlands, 1990.

[2] Bear J., Dynamics of Fluids in Porous Media, American Elsevier, New York, 1972.

[3] Boussinesq J., Comptes RendusAcad. science Journal, Math. Pures Appl., 10, 5-78, 1903.

[4] Darcy H., Les Fontaines publiques de loville de Dijon, Dalmont, Paris, 1856.

[5] Desai N. B. and Mehta M. N., A Solution of Seepage of ground water in soil: Homotopy Perturbation method. Int. J. of Appl. Math and Mech. 6 (2): 85-92, 2010.

[6] Haimson B., and Fairhurst C., J. Petrol. Technol. 21, 811-817, 1969.

[7] $\mathrm{He} \mathrm{JH}$, A coupling method of a homotopy technique and a perturbation technique for ono-lineat problems, Int. J. Non-linear Mech., 35:37-43, 2000.

[8] Horton Robert E., The role of infiltration in the hysrologic cycle., Trans. Am. Geophys. Un., 14th Ann. Mtg., 446-460, 1933.

[9] Liao S. J., A kind of approximate solution technique which does not depend upon small parameters (II): an application in fluid mechanics, Int. J. Nonlinear. Mech, 32-815, 1997.

[10] Liao S. J., An explicit, totally analytic approximation of Blasius viscous flow problems., Int. J. Nonlinear. Mech., Vol. 34, 759-778, 1999.

[11] Liao S. J., Beyond Perturbation: introduction to Homotopy Analysis Method. CRC Press: Chapman \& Hall; 2003.

[12] Liao S. J., Magyari E., Exponentially decaying boundary layers as limiting cases of families of algebraically decaying ones, ZAMP, 777-92, 57(5), 2006.

[13] Mehta M. N. and Meher R. K., Adomian decomposition method for moisture content in one dimensional fluid flow through unsaturated porous media, Int. J. of Appl. Math and Mech. 6(7): 13-23, 2010.

[14] Patel K. R., Mehta M. N. and Singh. T. R., A series solution of moisture content in vertical groundwater flow through unsaturated heterogeneous porous media, International eJournal of Mathematics and Engineering 159, 1467 - 1477, 2012.

[15] Patel T. and Mehta M. N., A solution of Burger's equation type one dimensional Ground water Recharge by spreading in Porous Media, Journal of Indian Acad. Math. 28(1), 25-32, 2006.

[16] Scheidegger A. E., The physics of flow through Porous media, Third Edition, University of Toronto Press, Toronto, 1974.

[17] Shapiro S. A., Dinske C., Geophys., Prospect., 57, 301-310, 2009.

[18] V'azquez J L., The Porous Medium equation- mathematical theory, Oxford science Publication, Clarden Press, Oxford, 2007. 